\title{
GABAergic basal forebrain projections to the periaqueductal gray promote food consumption, reward and predation
}

Ciorana Roman-Ortiz

Icahn School of Medicine at Mount Sinai

Jessica A. Guevara

St. Francis College

Roger L. Clem ( $\nabla$ roger.clem@mssm.edu )

Icahn School of Medicine at Mount Sinai

\section{Research Article}

Keywords: Overeating, Physiological Demand, Fictive Eating, Skilled Predatory Attack, Circuit Mechanism

Posted Date: June 10th, 2021

DOI: https://doi.org/10.21203/rs.3.rs-583865/v1

License: (c) (i) This work is licensed under a Creative Commons Attribution 4.0 International License.

Read Full License

Version of Record: A version of this preprint was published at Scientific Reports on November 22nd, 2021. See the published version at https://doi.org/10.1038/s41598-021-02157-7. 
1 GABAergic basal forebrain projections to the periaqueductal gray promote food

2 consumption, reward and predation

3 Ciorana Roman-Ortiz¹, Jessica A. Guevara², Roger L. Clem, ${ }^{1}$

$4{ }^{1}$ Nash Family Department of Neuroscience, Friedman Brain Institute, Icahn School of Medicine

5 at Mount Sinai, New York, NY ${ }^{2}$ St. Francis College, Department of Biological Sciences,

6 Brooklyn, NY

$7 \quad$ * Correspondence to roger.clem@mssm.edu.

9 ABSTRACT

10 Behaviors central to the procurement and consumption of food are among those most

11 fundamental to survival, but their inappropriate expression can lead to overeating and obesity.

12 Nevertheless, we have a poor understanding of circuits that promote feeding independent of

13 physiological demand. Here we demonstrate that activation of basal forebrain (BF) GABAergic

14 neurons results in consumption of food as well as non-food items in well-fed mice, and

15 performance of fictive eating in the absence of ingestible materials. In addition, stimulation of

16 these cells disrupts defensive threat responses and elicits reward-like motivational effects.

17 Finally, BF GABAergic activity triggers skilled predatory attack of live prey and prey-like objects,

18 but not social targets. These effects were entirely recapitulated by selective stimulation of BF

19 GABAergic projections to the periacqueductual gray (PAG). Our results outline a potent circuit

20 mechanism for increased feeding through recruitment of distinct but synergistic behaviors, and

21 add to growing evidence that PAG is an important integrator of feeding-related activity. 
Maladaptive overeating is one of the main causes of obesity, which in recent decades

26 has doubled in prevalence to an overwhelming $43 \%$ of U.S. adults (Hales and Ogden 2020).

27 Binge eating, which is characterized by recurrent periods of eating large amounts of food in the absence of physical hunger, is the most common eating disorder (Lehman 2000, Guerdjikova, Mori et al. 2019). Previous work in rodents has shed considerable light on the circuitry underlying homeostatic food consumption, which involves processing of neural signals for hunger and satiety by the lateral hypothalamus (LH) and arcuate nucleus (ARC) (Cowley, Smart et al. 2001, Chen, Lin et al. 2015, Jennings, Ung et al. 2015, Ferrario, Labouèbe et al. 2016, Stuber and Wise 2016). However, we have an incomplete understanding of neural pathways that modulate and/or coordinate more fundamental aspects of the feeding repertoire, the dysregulation of which could lead to food consumption even in the absence of homeostatic drive.

Although they have been primarily implicated in attention, arousal, and sleep/wake regulation (Avery, Dutt et al. 2014, Xu, Chung et al. 2015, Villano, Messina et al. 2017, Blake and Boccia 2018), recent reports suggest that neuronal populations in the basal forebrain (BF) contribute to food intake (Herman, Ortiz-Guzman et al. 2016, Cassidy, Lu et al. 2019). For example, activation of cholinergic as well as glutamatergic BF neurons decreases food consumption, whereas activation of GABAergic neurons increases food intake (Herman, OrtizGuzman et al. 2016, Zhu, Yao et al. 2017, Patel, Swanson et al. 2019, Cai, Chen et al. 2020). Moreover, BF GABAergic activity increases during naturally occurring food consumption and hunting (Cai, Chen et al. 2020). Despite the unique potential for BF GABAergic neurons to drive feeding, it remains unclear which aspects of behavior are primarily modulated by these cells or which downstream circuits underlie these responses. Among the areas receiving GABAergic projections from BF is the periaqueductal gray (PAG) (Do, Xu et al. 2016), a midbrain structure 
involved in critical survival-based processes including pain, defense, foraging and hunting (Behbehani 1995, De Oca, DeCola et al. 1998, Mota-Ortiz, Sukikara et al. 2009, Mota-Ortiz, F. et al. 2012). We therefore sought to establish the impact of BF GABAergic neurons, and their

52 PAG projections, on behaviors central to the procurement and consumption of food. We found 53 that a BF-PAG circuit promotes hunting and instrumental responding for food, as well as its 54 consumption, regardless of caloric value or existing homeostatic demands.

RESULTS

Activation of $\mathrm{BF}^{\mathrm{GAD2+}}$ neurons increases consummatory drive independent of caloric value.

In order to examine the behavioral role of BF GABAergic neurons in consummatory behaviors, we employed an optogenetic approach. First, we injected into basal forebrain of GAD2-Cre mice a Cre-dependent adeno-associated virus (AAV) expressing channelrhodopsin (ChR2) fused to an enhanced yellow fluorescent protein (AAV1-Ef1a-DIO-ChR2-eYFP) or an opsin-negative eYFP control vector (AAV1-Ef1a-DIO-eYFP), and implanted optic ferrules directed at the same site (Fig. 1a-b, Supplementary Fig.1). Because previous studies have reported that photoactivation of $\mathrm{BF}^{\mathrm{GAD2}+}$ neurons induces food consumption (Zhu, Yao et al. 2017, Cai, Chen et al. 2020), we first tested the effect of photostimulation paired with food

67 availability. The test chamber was divided into two zones, one of which contained food pellets.

68 Upon each entry to the food zone, photostimulation (473nm; $20 \mathrm{hz}, 10 \mathrm{~ms}$ pulses; $5-8 \mathrm{~mW}$ ) was 69 delivered for 60 seconds, which resulted in increased time spent in the food zone as well as 70 increased food intake (Fig. 1c-e). Given the pronounced effect on food consumption in well-fed 71 animals, we tested whether caloric value was a prerequisite for consumption by exposing the 72 animals to a willow tree branch and measuring the amount of wood removed by gnawing.

73 Animals that received $\mathrm{BF}^{\mathrm{GAD} 2+}$ activation gnawed more wood compared to eYFP control animals 
74 (Fig. 1f-g). Thus, activation of $\mathrm{BF}^{\mathrm{GAD2}+}$ neurons promotes consummatory behavior that is

directed at both food and non-food items, and therefore is independent of nutritional characteristics.

Given the apparent modulation of consummatory drive by $\mathrm{BF}^{\mathrm{GAD2+}}$ neurons, we next examined whether activation of these cells is sufficient to enhance performance in an appetitive operant task, in which animals are required to press an active lever for a food reward on a continuous reinforcement (FR1) schedule. Food-restricted mice received daily training sessions lasting 60 minutes or until they made 30 rewarded lever presses. Once animals met acquisition criteria (30 lever presses within 10 minutes), they were tested on the following day to determine the effect of $\mathrm{BF}^{\mathrm{GAD2+}}$ activation on established operant responding. Photostimulation had no discernible effect on lever pressing, indicating that increased consummatory drive did not facilitate a learned food-seeking behavior (Fig. 1h-i). Importantly, however, it also did not interfere with performance of this task.

Nevertheless, to determine whether $\mathrm{BF}^{\mathrm{GAD} 2+}$ activation has gross effects on exploration or cognition, we examined whether photostimulation alters locomotor activity and/or recognition memory in the novel object task. Animals were first familiarized to a novel object and, coinciding with photostimulation, a second novel object was introduced (Fig. 2a). During photostimulation, ChR2- versus eYFP-expressing animals did not differ in distance traveled within the arena (Fig. 2b). Likewise, photostimulation did not disrupt the preference to explore a novel versus familiar object, further indicating that it does not affect ongoing exploration, nor does it impair object perception or recognition memory in this task (Fig. 2c).

To determine whether $\mathrm{BF}^{\mathrm{GAD2} 2+}$ activation influences the expression of a negative valence association, we next examined the impact of photostimulation on cue-elicited freezing following auditory fear conditioning, which entailed 6 presentations of an auditory conditioned stimulus (CS, $2 \mathrm{kHz}, 90 \mathrm{~dB}, 20 \mathrm{~s}$ ) that co-terminated with an unconditioned stimulus (US, $0.7 \mathrm{~mA}$ foot shock, $2 \mathrm{~s}$ ) (Fig. 2d-e). When activation of $\mathrm{BF}^{\mathrm{GAD2} 2}$ neurons coincided with CS-evoked memory 
retrieval, freezing was markedly reduced compared to CS-only trials (Fig. 2f-g). Interestingly, during periods of reduced freezing, we noticed that animals were largely engaged in fictive eating behaviors (Fig. 2h), including licking and biting their surroundings, and raising empty paws to the mouth in a conventional feeding movement (Han, Tellez et al. 2017). In the absence of consumable objects, therefore, $\mathrm{BF}^{\mathrm{GAD} 2+}$ neurons initiate indiscriminate feeding movements and, surprisingly, imminent threat is not sufficient to suppress them.

Given the ability of $\mathrm{BF}^{\mathrm{GAD2} 2}$ neuron activation to override an aversive response, one possibility is that photostimulation of these cells induces a positive affective state. To test this hypothesis, we conducted an assay of real-time place preference (RT-PP), in which mice are allowed to freely explore two distinct chambers, one of which is paired with photostimulation (Fig. 3a). Compared to eYFP controls, ChR2-expressing mice spent far greater time exploring the photostimulation-paired chamber, consistent with a real-time preference for BFGAD2+ activation (Fig. 3b-d). However, this preference was not retained during subsequent testing in the absence of photostimulation, suggesting that with the given parameters, activation of $\mathrm{BF}^{\mathrm{GAD2+}}$ cell bodies does support the formation of a contextual reward association.

\section{Activation of $\mathrm{BF}^{\mathrm{GAD} 2+}$ neurons promotes predatory hunting.}

Broadly writ, feeding includes all actions integral to the procurement of nutrients. In many mammals, including mice, a critical aspect of feeding involves the pursuit and attack of prey, which is distinct from other forms of aggression. To determine whether $\mathrm{BF}^{\mathrm{GAD} 2+}$ neurons modulate these behaviors, we therefore examined the behavioral effects of photostimulating $\mathrm{BF}^{\mathrm{GAD2+}}$ neurons in the presence of crickets, which are natural prey of rodents. After habituation to both prey and experimental apparatus, we exposed well-fed mice to a single live cricket and quantified hunting-related behaviors including pursuit, subduction, and biting. During BFGAD2+ neuron activation, mice spent more time engaged in these behaviors, made more hunting attempts, and exhibited a decreased latency to initial attack (Fig. 4a-d, Supplementary Movie 1). 
Compared to ingestion, hunting involves relatively complex sensorimotor processing,

127 wherein the unique attributes of natural moving prey may be required to elicit and/or sustain

128 predator-prey interactions because they have been optimized by evolution for specific sensory

129 feedback. To test whether these behaviors depend on natural prey characteristics, we therefore

130 employed moving battery-powered toys (robobugs) as artificial prey-like objects (Fig. 4e).

131 Without exception, animals did not engage in hunting when exposed to robobugs under

132 baseline (light off) conditions. However, upon photoactivation of $\mathrm{BF}^{\mathrm{GAD2+}}{ }^{\text {neurons, we observed }}$

133 an increase in time spent hunting as well as the number of hunting attempts (Fig. 4f-g,

134 Supplementary Movie 2). Indeed, for the majority of ChR2-expressing mice, photostimulation 135 triggered immediate attack (Fig. 4h). To examine responses to an object that is more interactive than the robobug, which makes random movements, we next employed a remote-controlled toy car to which the animals were habituated beforehand to minimize novelty-related fear (Fig. 4i). During the test we purposely pursued the experimental animal with the car in order to force an interaction and, once an attack was initiated, escape was mimicked by retreating away from the subject. Under this scenario, we found that eYFP mice completely abstained from hunting, despite engaging in several investigative approaches (Fig. 4j-I, Supplementary Movie 3).

142 However, during light on ChR2-expressing mice spent far greater time hunting and, rather than 143 investigating the toy, they attacked it.

The above results suggest that $\mathrm{BF}^{\mathrm{GAD2} 2}$ neurons promote hunting regardless of whether 145 the target shares specific attributes of natural prey. Importantly, however, movement is a key 146 factor in prey detection, particularly in a large space such as that employed in our hunting 147 assays. Therefore, to test the impact of movement on hunting of natural and artificial prey, we 148 introduced immobilized crickets or robobugs. In both cases, we found that activation of BFGAD2+ 149 neurons failed to increase hunting and, instead, animals defaulted to fictive eating behaviors 150 during photostimulation (Supplementary Fig. 2). These data suggest that movement, and 151 potentially prey interaction, provide feedback essential for the predatory effects of BFGAD2+ 
activation. Although attacks are readily directed at moving objects, however, activation of $\mathrm{BF}^{\mathrm{GAD2+}}$ neurons did not trigger aggression toward novel conspecifics of either sex or otherwise affect social interaction in the home cage intruder test (Supplementary Fig. 3). Therefore, $\mathrm{BF}^{\mathrm{GAD2+}}$ neurons regulate a specific form of aggression that is integral to feeding and likely depends on a distinct underlying circuitry.

\section{$\mathrm{BF}^{\mathrm{GAD2+}} \rightarrow$ PAG projections mediate consumption, prey hunting and reward.}

Prior anatomical tracing has revealed extensive projections of basal forebrain GABAergic neurons throughout the brain (Do, Xu et al. 2016). Among their downstream targets are midbrain structures involved in aggression and feeding, such as the hypothalamus and PAG. A well-understood function of GABAergic projections arising from BF nuclei, including the bed nucleus of the stria terminalis (BNST), is the modulation of genetically-defined neuronal populations in the lateral hypothalamus (LH), which promotes food consumption and may be particularly important for hedonic feeding (Jennings, Rizzi et al. 2013, Jennings, Ung et al. 2015). Although LH is also involved in predation, evidence has more extensively implicated the PAG as a critical center for foraging and prey hunting, as well as defending against environmental threats encountered during these risky activities (Mota-Ortiz, Sukikara et al. 2009, Mota-Ortiz, F. et al. 2012, Hao, Yang et al. 2019, Rossier, La Franca et al. 2021). Examination of PAG confirmed the presence of dense projections from $\mathrm{BF}^{\mathrm{GAD2} 2}$ neurons that are particularly concentrated in the lateral and ventrolateral divisions (Fig. 5a-b), which have been specifically associated with predatory behavior (Comoli, Ribeiro-Barbosa et al. 2003). We therefore utilized projection-specific photoexcitation to test whether this pathway mediates effects of $\mathrm{BF}^{\mathrm{GAD2}}{ }^{+}$activity on prey hunting as well as other feeding behaviors.

Following infusion of an AAV-DIO-ChR2 vector into the basal forebrain of GAD2-Cre mice, we implanted an optic fiber directed at the PAG to enable selective stimulation of $\mathrm{BF}^{\mathrm{GAD} 2+} \rightarrow \mathrm{PAG}$ projections (Fig. 5a-b, Supplementary Fig. 4). Photostimulation (473 nm, $20 \mathrm{~Hz}$, 
$10 \mathrm{~ms}$ pulses, $5-8 \mathrm{~mW}$ ) of this pathway was sufficient to increase consumption of food pellets as well as time spent in the food-paired zone of a two-chamber arena (Fig. 5c-d). In addition, stimulation of ChR2-expressing mice but not eYFP controls promoted wood gnawing (Fig. 5e). Interestingly, activation of $\mathrm{BF}^{\mathrm{GAD} 2+} \rightarrow \mathrm{PAG}$ pathway also increased lever presses in the appetitive operant task, indicating that unlike stimulation of BF cell bodies (Fig. 1i), selective activation of this pathway facilitates food seeking behavior (Fig. 5f).

Following auditory fear conditioning, activation of $\mathrm{BF}^{\mathrm{GAD} 2+} \rightarrow$ projections recapitulated the impairment of CS-evoked freezing observed during cell body illumination (Fig. $5 \mathrm{~g}$ ) and this was likewise accompanied by the expression of fictive eating behaviors (Supplementary Fig. 5). In the test of place preference, photoexcitation resulted in a real-time preference for stimulationpaired chamber, consistent with reward-like reinforcement (Fig. 5h). In addition, in contrast to stimulation of $\mathrm{BF}^{\mathrm{GAD} 2+}$ cell bodies (Fig. $3 \mathrm{~d}$ ), $\mathrm{BF}^{\mathrm{GAD} 2+} \rightarrow \mathrm{PAG}$ activation resulted in robust conditioned place preference (CPP), which was expressed on the following day in the absence of photostimulation (Fig. 5i). These results indicate that $\mathrm{BF}^{\mathrm{GAD2}} \rightarrow \mathrm{PAG}$ projections can completely account for the consummatory effects of $\mathrm{BF}^{\mathrm{GAD} 2+}$ neurons and their selective activation is associated with stronger motivational effects in both Pavlovian and instrumental tasks.

Using an experimental design identical to that employed during $\mathrm{BF}^{\mathrm{GAD} 2+}$ cell body illumination, we then examined the potential for $\mathrm{BF}^{\mathrm{GAD} 2+} \rightarrow \mathrm{PAG}$ projections to modulate hunting of natural and artificial prey. During exposure to live crickets, photostimulation had no effect on hunting time but decreased the latency to attack (Fig. 5j-k). Similar to cell body illumination, $\mathrm{BF}^{\mathrm{GAD} 2+} \rightarrow \mathrm{PAG}$ activation also promoted hunting of moving artificial prey (robobugs) as well as an interactive toy car in animals that were not otherwise inclined to hunt these objects (Fig. 5lm). Therefore, a BF GABAergic pathway targeting the PAG supports fundamental components of the feeding repertoire, and promotes expression of stereotyped responses regardless of whether the target of these behaviors is suitable for consumption, let alone palatable. 
Recent studies have shown that BF cell types modulate feeding behaviors (Herman, 206 Ortiz-Guzman et al. 2016, Zhu, Yao et al. 2017, Patel, Swanson et al. 2019, Cai, Chen et al. 207 2020), and that GABAergic subtypes, in particular, promote the consumption of palatable food. 208 Here we demonstrate that consistent with these reports activation of BF GABAergic neurons 209 increases consumption but, importantly, this behavior is independent of the nutritional value. 210 Indeed, in the absence of ingestible material, stimulated animals engage in non-productive 211 fictive eating. Additionally, BF GABAergic activity induces hunting of natural prey as well as 212 prey-like electronic devices. These effects are recapitulated by selective stimulation of $\mathrm{BF} \rightarrow \mathrm{PAG}$ GABAergic projections, and accompanied by the loss of defensive threat responding, suggestive of a powerful motivational state that overrides competing survival-based demands. Modulation of feeding in well-fed animals establishes a functional parallel between BF GABAergic neurons and GABAergic projection cells of the LH and CeA (Navarro, Olney et al. 2016, Han, Tellez et al. 2017). Induction of real-time and conditioned place preference suggests

218 that stimulation of BF neurons and their GABAergic projections exerts a positive motivational 219 effect, which may in part explain the lack of discrimination between food and non-food items. On 220 the other hand, engagement in fictive eating during BF stimulation suggests the potential 221 recruitment of anatomical pathways impinging on the parvocellular reticular formation (PCRt), a 222 medullary premotor structure that controls oromotor and forelimb movements (Tellegen and 223 Dubbeldam 1999, Esposito, Capelli et al. 2014, Han, Tellez et al. 2017). While the PCRt is a 224 downstream target of the BF (Agostinelli, Geerling, and Scammell 2019), PAG neurons primarily 225 project to and modulate the mesencephalic locomotor region (Tovote, Esposito et al. 2016), 226 although sparse projections to the PCRt have been reported (Cameron, Khan et al. 1995). 227 Involvement of PCRt in BF $\rightarrow$ PAG effects is therefore likely to require an intermediary relay from 228 the PAG. One possibility is the CeA, which receives extensive PAG projections and in turn 
modulates orofacial movements via monosynaptic connections with PCRt (Do et al. 2016; Han et al. 2017).

A particularly intriguing effect of BF GABAergic activity was the disruption of conditioned fear. As in the case of consummatory behavior, this may in part be explained by the induction of a positive valence state that either neutralizes or overrides the aversive CS. Alternatively, superfluous orofacial movements may interfere with execution of freezing without affecting underlying fear. However, this explanation seems inadequate given that photostimulation did not impair exploratory locomotion, novel object recognition, operant responding or social interaction, which were successfully executed despite increased consummatory drive. Likewise, stimulation did not interfere with complex sensorimotor processing required for hunting. Indeed, suppression of fear by BF GABAergic neurons would be consistent with the hypothesized role of threat defense in constraining predation, and hence a requirement to override fear during the procurement of food (Pellman and Kim 2016, Rossier, La Franca et al. 2021). Because the PAG plays an integral role in both predation and defensive freezing (Mota-Ortiz, Sukikara et al. 2009, Mota-Ortiz, F. et al. 2012, Assareh, Sarrami et al. 2016, Tovote, Esposito et al. 2016) , further investigation of $\mathrm{BF}^{\mathrm{GAD} 2+} \rightarrow \mathrm{PAG}$ circuits may uncover novel, ethologically relevant insights into fear attenuation.

In this study, we found that stimulation of BF GABAergic neurons promoted skilled predatory hunting, as well as sustained attack of prey-like electronic devices. However, this was not observed in the case of immobilized prey, indicating that in addition to BF activity predation is facilitated by integration of specific sensory feedback. In addition, while animals readily engaged in hunting of moving artificial prey during photostimulation, they abstained from attacking a novel conspecific animal, indicating a selective involvement of $\mathrm{BF}^{\mathrm{GAD} 2+} \rightarrow \mathrm{PAG}$ projections in predatory but not social aggression. This further implies that perceptual 
discrimination is a prerequisite for target engagement upon activation of $B F \rightarrow$ PAG GABAergic 254 projections.

To a surprising degree, our data implicate GABAergic BF $\rightarrow$ PAG projections in several

256 distinct components of feeding, including consumption, reward and hunting. This stands in

257 contrast to a previous study in which $\mathrm{CeA} \rightarrow \mathrm{PAG}$ projections were found to exert selective

258 control over prey pursuit, but not consumption (Han, Tellez et al. 2017). This discrepancy may

259 be attributable to differences in the postsynaptic targeting of BF and CeA pathways, particularly

260 given that PAG has been independently implicated in food consumption (Hao, Yang et al.

261 2019). Evidence suggests that a common downstream effector of GABAergic populations in the

262 BNST and LH in consummatory behavior may be suppression of GABAergic neurons in the

263 ventrolateral PAG (Hao, Yang et al. 2019, Rossier, La Franca et al. 2021). Indeed, direct

264 suppression of ventrolateral PAG activity leads to food intake as well as long-term weight gain,

265 while inactivation of lateral PAG induces prey hunting (Han, Tellez et al. 2017, Li, Zeng et al.

266 2018, Hao, Yang et al. 2019, Zhao, Chen et al. 2019, Rossier, La Franca et al. 2021).

267 Meanwhile, afferents from the CeA and medial preoptic area (MPA) have also been shown to

268 modulate defensive freezing and predation, respectively (Tovote, Esposito et al. 2016, Park,

269 Jeong et al. 2018). Understanding how PAG encodes these fundamental and oftentimes

270 conflicting behavioral responses will require a more detailed understanding of its intrinsic

271 circuitry and afferent connectivity.

272

273 


\section{METHODS}

278 Animals: Adult GAD2-IRES-Cre male and female mice (P60-90) were maintained on a $12 \mathrm{~h}$

279 light-dark cycle with ad libitum access to food and water. Transgenic mice GAD2-IRES-Cre 280 (Stock No. 019022) were acquired from The Jackson Laboratory (Bar Harbor, ME, USA). All 281 experimental procedures were approved by the Institutional Animal Care and Use Committee at the Icahn School of Medicine at Mount Sinai, which is accredited by the Association for the Assessment and Accreditation of Laboratory Animal Care. All experiments were performed in accordance with relevant guidelines and regulations. All experiments are reported in accordance with the ARRIVE guidelines.

Stereotaxic virus injection and optic fiber implantation: Mice received bilateral infusion of AAV1-EF1a-DIO-hChR2 (H134R)-eYFP (Addgene 20298) or AAV1- Ef1a-DIO-eYFP (Addgene $27056)$ into basal forebrain (BF, encompassing ventral pallidum, substantia innominata and horizontal diagonal band; bregma coordinates $\mathrm{AP}+0.6, \mathrm{ML} \pm 1.6$, DV $-5.1-3$ ). Optic fibers ferrules $(200 \mu$ m diameter, Thorlabs) were placed over BF (AP +0.6, ML $\pm 1.6, \mathrm{DV}-5.1)$ or PAG (AP -4.36, ML $\pm 0.42, \mathrm{DV}-3.30$ ) and fixed with acrylic cement. Mice were allowed to recover for 7 days after surgery and habituated to experimenter handling for 3 consecutive days before 293 behavioral testing.

Behavioral testing: All experiments were carried out while animals were tethered to a patch cord to allow for laser light delivery. The order of light off and light on epochs was counterbalanced across all groups and experiments to control for any ordering effects of 297 photostimulation. All arenas were cleaned with Rescue ${ }^{\mathrm{TM}}$ disinfectant (Oakville, ON, USA) after 298 each animal to eliminate any odor cues. Video recording of each test was performed using Any299 maze behavioral tracking software (Version 4.99, Stoelting Co., Wood Dale, IL, USA) unless 300 otherwise specified in the sections below. Hunting behaviors as well as social interaction was 301 manually scored by a trained observer naïve to experimental groups. 
Food paired photostimulation: For measurement of food consumption, mice were preexposed to dustless food pellets (45mg, BIO-SERV, Flemingtown, NJ, USA) in their home cage for at least 3 days prior to behavioral testing. Fed (ad libitum) mice were placed in a behavioral box (L: $29.21 \mathrm{~cm} \times$ W: $19.05 \mathrm{~cm} \times \mathrm{H}: 16.51 \mathrm{~cm}$ ) divided into two zones; a food zone (with a container of food pellets) and a no food zone (which was empty). The food zone was paired with light stimulation (473 nm; $20 \mathrm{~Hz}, 10$ ms pulses; $5-8 \mathrm{~mW}$ ) for 60 seconds upon entry. Animals were allowed move between the two zones for 20 minutes, and time spent in each zone was quantified using Any-maze behavioral tracking software (Version 4.99, Stoelting Co., Wood Dale, IL, USA).

Wood gnawing test: For the wood gnawing assay, we placed a willow tree branch $(7.62 \mathrm{~cm})$ in a clean empty behavioral arena ( $\mathrm{L}: 29.21 \mathrm{~cm} \times \mathrm{W}: 19.05 \mathrm{~cm} \times \mathrm{H}: 16.51 \mathrm{~cm})$ and then placed the mice in the arena. The test consisted of two epochs (20 minutes each), one of which was paired with laser stimulation (473 nm; $20 \mathrm{~Hz}, 10 \mathrm{~ms}$ pulses; $5-8 \mathrm{~mW}$ ) in a counterbalanced order. Between each epoch the cage was cleaned with Rescue ${ }^{T M}$ disinfectant (Oakville, ON, USA), and the weight of the branch was obtained.

Cricket hunting: In order to familiarize the experimental mice with crickets, we placed the mice in a behavioral box with 3 crickets for 30 minutes on each of three days prior to the hunting assay. On the day of the assay, fed (ad libitum) or fasted (12h, dark cycle) mice were placed in a test arena (L: $40 \mathrm{~cm} \times \mathrm{W}: 20 \mathrm{~cm} \times \mathrm{H}: 25 \mathrm{~cm}$ ) allowed to habituate for 10 minutes. The cage was then cleaned with Rescue ${ }^{\mathrm{TM}}$ disinfectant (Oakville, ON, USA), and to start each trial the mouse was placed in one corner of the cage and the cricket (juvenile, medium-sized) was released in the opposite corner. Each trial lasted 10 minutes and involved a fresh (unharmed) cricket. We performed four trials for each mouse, alternating between laser off and laser on (473 $\mathrm{nm} ; 20 \mathrm{~Hz}, 10 \mathrm{~ms}$ pulses; $5-8 \mathrm{~mW}$ ) trials, with the order of photostimulation being counterbalanced across animals. For the static prey assay dead crickets were used in place of 
327 live ones. The trial videos were manually scored for hunting behaviors by a trained observer 328 blind to experimental groups.

329 Artificial prey hunting: Mice were placed in a clean empty cage (L: $39.1 \mathrm{~cm} \mathrm{x} \mathrm{W:19.9} \mathrm{cm} \mathrm{x}$ $\mathrm{H}: 16 \mathrm{~cm}$ ) with artificial moving prey (miniature $(3.81 \mathrm{~cm})$ battery-powered toy, HEXBUG® Nano, Amazon.com) for two epochs that lasted 2 minutes each, similar to the hunting assay used in 332 (Han, Tellez et al. 2017). To assay hunting of the static toy, we used the same design with the exception that the HEXBUG ${ }^{\circledR}$ was switched off and therefore not moving. Laser stimulation (473 $\mathrm{nm} ; 20 \mathrm{~Hz}, 10 \mathrm{~ms}$ pulses; $5-8 \mathrm{~mW}$ ) was paired with one of the two epochs, with the order of photostimulation being counterbalanced. Hunting behaviors were manually scored by a trained observer naive to experimental conditions.

Interactive toy test: To facilitate interaction between the experimental mice and prospective artificial prey we used a remote-controlled toy car (Arris Mini RC Car, Amazon.com, L: $6.7 \mathrm{~cm} \mathrm{x}$ $\mathrm{W}: 2 \mathrm{~cm} \times \mathrm{H}: 2.8 \mathrm{~cm}$ ) that was guided by the experimenter. One day prior to the test mice were first habituated to the toy for 30 minutes in the test arena ( $\mathrm{L}: 40 \mathrm{~cm} \times \mathrm{W}: 20 \mathrm{~cm} \times \mathrm{H}: 25 \mathrm{~cm})$. The test consisted of two epochs (2 minutes each), alternating between laser off and laser on (473 $\mathrm{nm} ; 20 \mathrm{~Hz}, 10 \mathrm{~ms}$ pulses; 5-8 mW), with the order of photostimulation being counterbalanced. Hunting behaviors were manually scored by a trained observer blind to experimental conditions.

Real-time and conditioned place preference: Real-time place preference (RT-PP) was performed in a rectangular box consisting of three compartments: two side chambers (L: $28 \mathrm{~cm}$ $\times \mathrm{W}: 24 \mathrm{~cm}$ each) connected by a center chamber (L: $11.5 \mathrm{~cm} \times \mathrm{W}: 24 \mathrm{~cm})$. Each compartment 347 has a distinct tactile (small grid vs. bars vs. large grid flooring) and visual (grey vs. black vs. stripped walls) cues to create different environments. One day prior testing, mice were allowed to explore the entire apparatus for 30 minutes (habituation). On the day of the test, one side 350 chamber was paired with laser light ( $473 \mathrm{~nm} ; 20 \mathrm{~Hz}, 10 \mathrm{~ms}$ pulses; 5-8 mW) beginning upon 351 entry. The test lasted 20 minutes. Chamber pairings were counterbalanced across animals. On 
352 the next day, during the test of conditioned place preference (CPP; 5 minutes), mice were again 353 allowed to freely explore the entire apparatus with no laser light presented. Mice location was 354 tracked and quantify with Any-maze behavioral tracking software (Version 4.99, Stoelting Co., 355 Wood Dale, IL, USA).

356 Operant appetitive task: Mice were allowed to acclimate to the behavior room for 30 minutes before each session. Prior to the first training session the animals were fasted (12h, dark cycle) and subsequently maintained on a food restricted diet across training. Operant food reinforcement was performed in standard operant chambers (Model MED-307W-D1; Med Associates, Fairfax, VT, USA) equipped with 2 retractable levers (active and inactive). The active lever was defined as the lever that upon pressing results in delivery of a chocolate flavored pellet (45mg, BIO-SERV, Flemingtown, NJ, USA) in a fixed ratio 1 (FR1) schedule (one-to-one), while inactive lever presses resulted in no programed reward. Active lever identity was counter-balanced across all animals. Each training session was terminated when the mice reached a maximum of 30 pellets or when the training session reached 60 minutes, whichever occurred first. The mice were trained for one session per day until they achieved 30 active lever presses during the first 10 minutes of the sessions ( $\sim 3-5$ sessions). Once the animals met acquisition criteria, a test lasting 20 minutes, with no maximum reward, was administered on the following day. The test had two epochs: one with laser off and one with laser on $(473 \mathrm{~nm} ; 20 \mathrm{~Hz}$, $10 \mathrm{~ms}$ pulses; 5-8 mW), with the order of photostimulation being counterbalanced. Lever presses (active and inactive) were obtained using Med-PC V Software Suite (Fairfax, VT, USA).

372 Novel object recognition: The test consisted of three phases; habituation (5 minutes), Object 3731 exploration (familiarization, 10 minutes), and object 1 vs object 2 (familiar object vs. novel 374 object, 10 minutes). During the habituation phase the mice were able to freely explore the empty 375 arena $(42 \mathrm{~cm} \times \mathrm{W}: 42 \mathrm{~cm} \times \mathrm{H}: 30 \mathrm{~cm})$, then at the beginning of the familiarization phase a small 376 object is placed into the arena, and lastly, during the last phase a second distinct object is 
377 introduced. The objects used for the test varied in texture and color to allow for differentiation.

378 Laser light (473 nm; $20 \mathrm{~Hz}, 10 \mathrm{~ms}$ pulses; 5-8 mW) was only present in the last phase (familiar 379 object vs. novel object), and object exploration time and distance traveled was quantified with 380 Any-maze behavioral tracking software (Version 4.99, Stoelting Co., Wood Dale, IL, USA). The 381 discrimination index was calculated as follows; time spend exploring novel object - time spent 382 exploring familiar object/ total time spent exploring novel and familiar objects.

383 Fear conditioning: Fear conditioning and memory retrieval were performed as describe in 384 (Cummings and Clem 2020). Briefly, mice underwent fear conditioning consisting six pairings of 385 an auditory tone $(2 \mathrm{kHz}, 90 \mathrm{~dB}, 20 \mathrm{~s})$ with a co-terminating foot shock $(0.7 \mathrm{~mA}, 2 \mathrm{~s})$. The test of fear memory retrieval consisted of four tone presentations in a different context (Context B), and 387 three laser light presentations ( $473 \mathrm{~nm} ; 20 \mathrm{~Hz}, 10 \mathrm{~ms}$ pulses; $5-8 \mathrm{~mW}$ for 20 seconds). Two of 388 the tone presentations will be paired with laser light in an alternated fashion. Freezing and fictive eating was manually scored by a trained observer blind to experimental conditions from videos recorded using Video Freeze® software (Med Associates, Fairfax, VT, USA).

391 Intruder test: Experimental mice were allowed to freely explore a new home cage (L: $39.1 \mathrm{~cm} \mathrm{x}$ $392 \mathrm{~W}: 19.9 \mathrm{~cm} \times \mathrm{H}: 16 \mathrm{~cm}$ ) for $5 \mathrm{~min}$ (habituation), before introduction of an age-matched novel 393 conspecific (female or male). Social interaction was then monitored during two contiguous 394 epochs (lasting 3 minutes each), one of which was paired with photostimulation (473 nm; $20 \mathrm{~Hz}$, $39510 \mathrm{~ms}$ pulses; 5-8 mW, $3 \mathrm{~min}$ ) in a counterbalanced order. Time interacting was manually 396 scored by a trained observer blind to experimental conditions.

Data analysis: Before inclusion of individual animals, both viral expression and fiber placement within the BF were histologically confirmed. Normality was assessed using a Shapiro-Wilk test 399 of individual group data as well as residual values of parametric comparisons, where applicable. 400 Parametric comparisons (i.e. paired or unpaired t-tests) were used only when normality was 401 supported under both conditions. Otherwise, non-parametric comparisons (i.e. Mann-Whitney, 
402 Wilcoxon signed ranked test) were used. Because data were generally non-Gaussian, we did 403 not test for interactions between independent variables (e.g. using two-way ANOVA). Statistical 404 analysis and graphing were performed in Prism 9 (Graphpad; La Jolla, CA, USA). Statistical 405 significance was set at $\mathrm{P}<0.05$ for all parametric and non-parametric tests and only 406 comparisons with $\mathrm{P}<0.05$ are explicitly reported. Detailed statistics are provided in figure 407 legends. Figure data are expressed in the form of box and whisker plots overlayed with 408 individual data points. Box and whisker plots are constructed as follows: line = median; boxes = $40925^{\text {th }}-75^{\text {th }}$ percentiles; whiskers $=$ minimum and maximum values. Behavioral diagrams were 410 created using BioRender. 
425

426

427

428

429

430

Assareh, N., M. Sarrami, P. Carrive and G. P. McNally (2016). "The organization of defensive behavior elicited by optogenetic excitation of rat lateral or ventrolateral periaqueductal gray." Behav Neurosci 130(4): 406-414.

Avery, M. C., N. Dutt and J. L. Krichmar (2014). "Mechanisms underlying the basal forebrain enhancement of top-down and bottom-up attention." European Journal of Neuroscience 39(5): 852-865.

Behbehani, M. M. (1995). "Functional characteristics of the midbrain periaqueductal gray." Prog Neurobiol 46(6): 575-605.

Blake, M. G. and M. M. Boccia (2018). "Basal Forebrain Cholinergic System and Memory." Curr Top Behav Neurosci 37(1866-3370 (Print)): 253-273.

Cai, P., L. Chen, Y. R. Guo, J. Yao, H. Y. Chen, Y. P. Lu, S. N. Huang, P. He, Z. H. Zheng, J. Y. Liu, J. Chen, L. H. Hu, S. Y. Chen, L. T. Huang, G. Q. Chen, W. T. Tang, W. K. Su, H. Y. Li, W. X. Wang and C. X. Yu (2020). "Basal forebrain GABAergic neurons promote arousal and predatory hunting." (1873-7064 (Electronic)).

Cameron, A. A., I. A. Khan, K. N. Westlund and W. D. Willis (1995). "The efferent projections of the periaqueductal gray in the rat: A Phaseolus vulgaris-leucoagglutinin study. II. Descending projections." Journal of Comparative Neurology 351(4): 585-601.

Cassidy, R. M., Y. Lu, M. Jere, J.-B. Tian, Y. Xu, L. R. Mangieri, B. Felix-Okoroji, J. Selever, Y. Xu, B. R. Arenkiel and Q. Tong (2019). "A lateral hypothalamus to basal forebrain neurocircuit promotes feeding by suppressing responses to anxiogenic environmental cues." Science Advances 5(3): eaav1640.

Chen, Y., Y. C. Lin, T. W. Kuo and Z. A. Knight (2015). "Sensory detection of food rapidly modulates arcuate feeding circuits." Cell 160(5): 829-841.

Comoli, E., E. R. Ribeiro-Barbosa and N. S. Canteras (2003). "Predatory hunting and exposure to a live predator induce opposite patterns of Fos immunoreactivity in the PAG." Behavioural Brain Research 138(1): 17-28.

Cowley, M. A., J. L. Smart, M. Rubinstein, M. G. Cerdán, S. Diano, T. L. Horvath, R. D. Cone and M. J. Low (2001). "Leptin activates anorexigenic POMC neurons through a neural network in the arcuate nucleus." Nature 411(6836): 480-484.

Cummings, K. A. and R. L. Clem (2020). "Prefrontal somatostatin interneurons encode fear memory." Nature Neuroscience 23(1): 61-74.

De Oca, B. M., J. P. DeCola, S. Maren and M. S. Fanselow (1998). "Distinct regions of the periaqueductal gray are involved in the acquisition and expression of defensive responses." J.Neurosci. 18(9): 34263432.

Do, J. P., M. Xu, S.-H. Lee, W.-C. Chang, S. Zhang, S. Chung, T. J. Yung, J. L. Fan, K. Miyamichi, L. Luo and Y. Dan (2016). "Cell type-specific long-range connections of basal forebrain circuit." eLife 5: e13214.

Esposito, M. S., P. Capelli and S. Arber (2014). "Brainstem nucleus MdV mediates skilled forelimb motor tasks." Nature 508(7496): 351-356.

Ferrario, C. R., G. Labouèbe, S. Liu, E. H. Nieh, V. H. Routh, S. Xu and E. C. Connor (2016). "Homeostasis Meets Motivation in the Battle to Control Food Intake." The Journal of Neuroscience 36(45): 11469.

Guerdjikova, A. I., N. Mori, L. S. Casuto and S. L. McElroy (2019). "Update on Binge Eating Disorder." Med Clin North Am 103(4): 669-680. 
Hales, C. M., Carroll, M.D., Fryar C.D., \& and C. Ogden (2020). "Prevalence of Obesity and Severe Obesity Among Adults: United States, 2017-2018." (1941-4927 (Electronic)).

Han, W., L. A. Tellez, M. J. Rangel, S. C. Motta, X. Zhang, I. O. Perez, N. S. Canteras, S. J. ShammahLagnado, A. N. van den Pol and I. E. de Araujo (2017). "Integrated Control of Predatory Hunting by the Central Nucleus of the Amygdala." Cell 168(1): 311-324.e318.

Hao, S., H. Yang, X. Wang, Y. He, H. Xu, X. Wu, L. Pan, Y. Liu, H. Lou, H. Xu, H. Ma, W. Xi, Y. Zhou, S. Duan and $\mathrm{H}$. Wang (2019). "The Lateral Hypothalamic and BNST GABAergic Projections to the Anterior Ventrolateral Periaqueductal Gray Regulate Feeding." Cell Rep 28(3): 616-624 e615.

Herman, A. M., J. Ortiz-Guzman, M. Kochukov, I. Herman, K. B. Quast, J. M. Patel, B. Tepe, J. C. Carlson, K. Ung, J. Selever, Q. Tong and B. R. Arenkiel (2016). "A cholinergic basal forebrain feeding circuit modulates appetite suppression." Nature 538(7624): 253-256.

Jennings, J. H., G. Rizzi, A. M. Stamatakis, R. L. Ung and G. D. Stuber (2013). "The inhibitory circuit architecture of the lateral hypothalamus orchestrates feeding." Science (New York, N.Y.) 341(6153): 1517-1521.

Jennings, Joshua H., Randall L. Ung, Shanna L. Resendez, Alice M. Stamatakis, Johnathon G. Taylor, J. Huang, K. Veleta, Pranish A. Kantak, M. Aita, K. Shilling-Scrivo, C. Ramakrishnan, K. Deisseroth, S. Otte and Garret D. Stuber (2015). "Visualizing Hypothalamic Network Dynamics for Appetitive and Consummatory Behaviors." Cell 160(3): 516-527.

Lehman, J. F. (2000). "The diagnostic and statistical manual of mental disorders."

Li, Y., J. Zeng, J. Zhang, C. Yue, W. Zhong, Z. Liu, Q. Feng and M. Luo (2018). "Hypothalamic Circuits for Predation and Evasion." Neuron 97(4): 911-924.e915.

Mota-Ortiz, S. R., S. M. F., B. J. C., B. M. V, E. C. F., F. L. F. and N. S. Canteras (2012). "The periaqueductal gray as a critical site to mediate reward seeking during predatory hunting." Behavioural Brain Research 226(1872-7549 (Electronic)): 32-40.

Mota-Ortiz, S. R., M. H. Sukikara, L. F. Felicio and N. S. Canteras (2009). "Afferent connections to the rostrolateral part of the periaqueductal gray: a critical region influencing the motivation drive to hunt and forage." Neural Plast 2009(1687-5443 (Electronic)): 612698.

Navarro, M., J. J. Olney, N. W. Burnham, C. M. Mazzone, E. G. Lowery-Gionta, K. E. Pleil, T. L. Kash and T. E. Thiele (2016). "Lateral Hypothalamus GABAergic Neurons Modulate Consummatory Behaviors Regardless of the Caloric Content or Biological Relevance of the Consumed Stimuli." Neuropsychopharmacology 41(6): 1505-1512.

Park, S.-G., Y.-C. Jeong, D.-G. Kim, M.-H. Lee, A. Shin, G. Park, J. Ryoo, J. Hong, S. Bae, C.-H. Kim, P.-S. Lee and D. Kim (2018). "Medial preoptic circuit induces hunting-like actions to target objects and prey." Nature Neuroscience 21(3): 364-372.

Patel, J. M., J. Swanson, K. Ung, A. Herman, E. Hanson, J. Ortiz-Guzman, J. Selever, Q. Tong and B. R. Arenkiel (2019). "Sensory perception drives food avoidance through excitatory basal forebrain circuits." eLife 8: e44548.

Pellman, B. A. and J. J. Kim (2016). "What Can Ethobehavioral Studies Tell Us about the Brain's Fear System?" Trends Neurosci 39(6): 420-431.

Rossier, D., V. La Franca, T. Salemi, S. Natale and C. T. Gross (2021). "A neural circuit for competing approach and defense underlying prey capture." Proceedings of the National Academy of Sciences 118(15): e2013411118. 
Stuber, G. D. and R. A. Wise (2016). "Lateral hypothalamic circuits for feeding and reward." Nat Neurosci 19(2): 198-205.

Tellegen, A. J. and J. L. Dubbeldam (1999). "Location of reticular premotor areas of a motor center innervating craniocervical muscles in the mallard (Anas platyrhynchos L.)." Journal of Comparative Neurology 405(3): 281-298.

Tovote, P., M. S. Esposito, P. Botta, F. Chaudun, J. P. Fadok, M. Markovic, S. B. Wolff, C. Ramakrishnan, L. Fenno, K. Deisseroth, C. Herry, S. Arber and A. Luthi (2016). "Midbrain circuits for defensive behaviour." Nature 534(7606): 206-212.

Villano, I., A. Messina, A. Valenzano, F. Moscatelli, T. Esposito, V. Monda, M. Esposito, F. Precenzano, M. Carotenuto, A. Viggiano, S. Chieffi, G. Cibelli, M. Monda and G. Messina (2017). "Basal Forebrain Cholinergic System and Orexin Neurons: Effects on Attention." Frontiers in Behavioral Neuroscience 11: 10.

Xu, M., S. Chung, S. Zhang, P. Zhong, C. Ma, W.-C. Chang, B. Weissbourd, N. Sakai, L. Luo, S. Nishino and Y. Dan (2015). "Basal forebrain circuit for sleep-wake control." Nat Neurosci 18(11): 1641-1647.

Zhao, Z.-d., Z. Chen, X. Xiang, M. Hu, H. Xie, X. Jia, F. Cai, Y. Cui, Z. Chen, L. Qian, J. Liu, C. Shang, Y. Yang, X. Ni, W. Sun, J. Hu, P. Cao, H. Li and W. L. Shen (2019). "Zona incerta GABAergic neurons integrate preyrelated sensory signals and induce an appetitive drive to promote hunting." Nature Neuroscience 22(6): 921-932.

Zhu, C., Y. Yao, Y. Xiong, M. Cheng, J. Chen, R. Zhao, F. Liao, R. Shi and S. Song (2017). "Somatostatin Neurons in the Basal Forebrain Promote High-Calorie Food Intake." Cell Reports 20(1): 112-123.

(1)

(1)

8


537 We thank F.A. Cinque, S. Bayshtok and A. Khawaja for their technical assistance. This research

538 was supported by the National Institute of Mental Health RO1 MH105414, RO1 MH116145 and

539 R21 MH114170 to R.L.C, and the National Institute of Neurological Disorders and Stroke F99

$540 \quad$ NS113458 to C.R.O.

541

542 COMPETING INTERESTS

543 The authors declare no competing interests.

544

545 CORRESPONDING AUTHOR

546 Roger L. Clem

547 Email: roger.clem@mssm.edu

548

549 AUTHOR CONTRIBUTIONS

550 C.R.O. and R.L.C. initiated the project. C.R.O and R.L.C. designed the experiments. C.R.O. and

551 J.A.G. performed the experiments and collected data. C.R.O. performed all the data analysis.

552 R.L.C. and C.R.O. wrote the manuscript.

553

554

555

556 
558 Figure 1. Activation of $\mathrm{BF}^{\mathrm{GAD2+}}$ neurons increases consummatory drive.

559 a, Stereotaxic targeting of viral vectors and optogenetic stimulation. b, Confocal image of viral 560 expression and optic fiber placement in BF, $200 \mu \mathrm{m}$ scale bar. c, Heatmap of center body location, 561 mean of eYFP and ChR2 groups, during test of food exposure paired with optic stimulation. One side of the arena contained food pellets, whereupon entry triggered optic stimulation. $\mathbf{d}$, Percent time spent in food zone. Mann-Whitney test, $U=36,{ }^{* * *} p<0.001$. ChR2, $n=15 ;$ eYFP, $n=17$. e,

564 Food intake, ingested mass. Mann-Whitney test, $U=54.5,{ }^{* *} p<0.01$. ChR2, $n=15 ;$ eYFP $n=17$.

f. Design for test of wood gnawing behavior, wherein animals were exposed to a willow tree branch with and without laser stimulation, in a counterbalanced fashion. g, Amount of wood

567 removed due to gnawing during laser ${ }^{\mathrm{ON}}$ vs laser ${ }^{\mathrm{OFF}}$ epochs. ChR2, Wilcoxon signed rank test, $W$ $568=44,{ }^{*} p<0.05$. ChR2, $n=11$; eYFP, $n=12 . h$, Design of test for operant responding for food. 569 Task entails discrimination between two levers, only one of which upon pressing will result in food 570 delivery. i, Active lever presses during laser ${ }^{\mathrm{ON}}$ vs laser ${ }^{\mathrm{OFF}}$ epochs. ChR2, n= 6; eYFP, n= 6.

572 Figure 2. $\mathrm{BF}^{\mathrm{GAD2+}}$ neuron activation does not affect exploration or object recognition but 573 disrupts conditioned fear expression.

574 a, Design of novel object recognition test. $\mathbf{b}$, Discrimination index is measured as the time spent 575 exploring the novel object - time spent exploring the familiar object divided by the total time 576 exploring novel object and familiar object. ChR2, n= 12; eYFP, $n=17$. c, Total distance traveled 577 during laser ${ }^{\mathrm{N}}$ epoch. ChR2, n= 12; eYFP, $n=17 . d$, Design for test of fear memory expression. 578 Following conditioning in context A, animals were placed into a distinct arena (context B) and 579 presented with 4 CS trials, two of which coincided with photostimulation, in a counterbalanced 580 fashion. e, Freezing levels during final two CS trials of conditioning. f, CS-evoked freezing during 581 laser ${ }^{\mathrm{ON}}$ vs. laserOFF CS trials. ChR2, Wilcoxon signed rank test, $W=-66,{ }^{* *} \mathrm{p}<0.01$. eYFP, paired 
582

t-test, $\mathrm{t}_{10}=2.40,{ }^{*} \mathrm{p}<0.05 . \mathrm{ChR2}, \mathrm{n}=12 ;$ eYFP, $\mathrm{n}=11 . \mathrm{g}$, Difference in freezing during laser ${ }^{\mathrm{ON}}$ vs. laser ${ }^{\mathrm{OFF}} \mathrm{CS}$ trials. ChR2, $\mathrm{n}=12$; eYFP, $\mathrm{n}=11$. $\mathbf{h}$, Fictive eating during laser ${ }^{\mathrm{ON}}$ vs. laser ${ }^{\mathrm{OFF}} \mathrm{CS}$ trials. ChR2, Wilcoxon signed rank test, $W=45,{ }^{* *} p<0.01$.

\section{Figure 3. Reward-like effect of $\mathrm{BF}^{\mathrm{GAD} 2+}$ activation.}

a, Design for test of real-time and conditioned place preference. b, Heatmap of center body location, of ChR2 and eYFP sample animal. ChR2, $\mathrm{n}=10$; eYFP, $\mathrm{n}=8$. c, Real-time place preference, measured on day 1 during photostimulation. Percent time in unpaired side, MannWhitney test, $U=12,{ }^{*} p<0.05$. Percent time in stimulation paired side, Mann-Whitney test, $U=$ 10, ${ }^{* *} p<0.01$. ChR2, $\mathrm{n}=10 ;$ eYFP, $\mathrm{n}=8 . \mathbf{d}$, Conditioned place preference, measured on day 2 in the absence of photostimulation. ChR2, $n=10 ;$ eYFP, $n=8$.

\section{Figure 4. $\mathrm{BF}^{\mathrm{GAD2+}}$ activation promotes hunting of live and artificial prey.}

a, Design of live cricket prey hunting assay used in b-d. b, Percent time hunting, defined as the time spent pursuing and capturing the cricket. ChR2, Wilcoxon signed rank test, $W=43,{ }^{*} p<$ 0.05. ChR2, $n=11$; eYFP, $n=11$. c, Number of hunting attempts. ChR2, paired t-test, $t_{10}=2.36$, ${ }^{*} p<0.05$. ChR2, $n=11$, eYFP, $n=11$. d, Latency to attack. ChR2, Wilcoxon signed rank test, $W$ $=-43,{ }^{*} p<0.05$. e, Design for artificial prey hunting assay used in f-h. Artificial prey consisted of a small toy (robobug) that is propelled by vibration. f, Percent time hunting. ChR2, Wilcoxon signed rank test, $W=45,{ }^{* *} p<0.01$. ChR2, $\mathrm{n}=9$; eYFP, $\mathrm{n}=14$. $\mathrm{g}$, Number of hunting attempts. ChR2, Wilcoxon signed rank test, $W=45,{ }^{* *} p<0.01$. h, Latency to attack. ChR2, Wilcoxon signed rank test, $W=45,{ }^{* *} p<0.01$. i, Design of the interactive artificial prey assay used in $\mathbf{j}-\mathbf{I}$. Interactive prey consisted of a toy car remote-controlled by the experimenter. j, Percent time hunting. ChR2, Wilcoxon signed rank test, $W=45,{ }^{* *} \mathrm{p}<0.01$. ChR2, $\mathrm{n}=9$; eYFP, $\mathrm{n}=7 . \mathbf{k}$, Number of hunting attempts. ChR2, Wilcoxon signed rank test, $W=45,{ }^{* *} p<0.01$. ChR2, $\mathrm{n}=9$; 
607

608

609

610

eYFP, $\mathrm{n}=7$. I, Latency to attack. ChR2, Wilcoxon signed rank test, $W=45,{ }^{* *} p<0.01$. ChR2, $\mathrm{n}$ = 9; eYFP, $\mathrm{n}=7$.

Figure 5. PAG projections mediate BF effects on consumption, reinforcement and predatory hunting.

a, Stereotaxic targeting of viral vectors and optogenetic stimulation. $\mathbf{b}$, Confocal image of viral expression and optic fiber placement in BF, $200 \mu \mathrm{m}$ scale bar. c, Percent time spent in food zone during optic stimulation paired with food. Mann-Whitney test, $U=14,{ }^{*} p<0.05$. ChR2, $n=8$; eYFP, $n=10 . d$, Food intake, ingested mass. Unpaired t-test, $t_{16}=3.66 .{ }^{* *} p<0.01$. ChR2, $n=$ 8; eYFP, $n=10$. e, Amount of wood removed due to gnawing during laser ${ }^{\mathrm{ON}}$ vs laser ${ }^{\mathrm{OFF}}$ epochs. ChR2, paired t-test, $t_{6}=2.38$. ChR2, $n=7$; eYFP, $n=10 . \mathbf{f}$, Active lever presses during test of operant responding for food. ChR2, paired t-test, $t_{6}=3.78,{ }^{*} p<0.05$. ChR2, $n=7 ;$ eYFP, $n=5$. g, CS-evoked freezing during laser ${ }^{\mathrm{ON}}$ vs. laser ${ }^{\mathrm{OFF}} \mathrm{CS}$ trials, following the experimental design in Fig. 2d. ChR2, Wilcoxon signed rank test, $W=-36,{ }^{* *} p<0.01$. ChR2, $n=8$; eYFP, $n=7 . \mathbf{h}$, Realtime place preference, measured on day 1 during photostimulation. Percent time in unpaired side, unpaired t-test, $\mathrm{t}_{12}=6.03,{ }^{* * * *} \mathrm{p}<0.0001$. Percent time in stimulation paired side, paired t-test, $\mathrm{t}_{12}=6.06,{ }^{* * * *} \mathrm{p}<0.0001 . \mathrm{ChR} 2, \mathrm{n}=8$; eYFP, $\mathrm{n}=6$. i, Conditioned place preference, measured on day 2 in the absence of photostimulation. Percent time in unpaired side, paired t-test, t12 = 3.62, ${ }^{* *} p<0.01$. Percent time in stimulation paired side, paired t-test, $t_{12}=3.62,{ }^{* *} p<0.01$. ChR2, $n=8$; eYFP, $n=6$. j, Percent time spent hunting in the live cricket assay. ChR2, $n=9$; eYFP, $n=10$. $\mathbf{k}$, Latency to attack in the live cricket assay. ChR2, paired t-test, $t_{8}=2.82,{ }^{*} p<$ 0.05. ChR2, $n=9$; eYFP, $n=10$. I, Percent time hunting in the artificial prey assay. ChR2, Wilcoxon signed rank test, $W=36,{ }^{* *} p<0.01$. ChR2, $n=8$; eYFP, $n=9 . \mathbf{m}$, Percent time hunting in the interactive artificial prey assay. ChR2, Wilcoxon signed rank test, $W=36,{ }^{* *} p<0.01$. ChR2, $\mathrm{n}=8 ;$ eYFP, $\mathrm{n}=9$ 
a

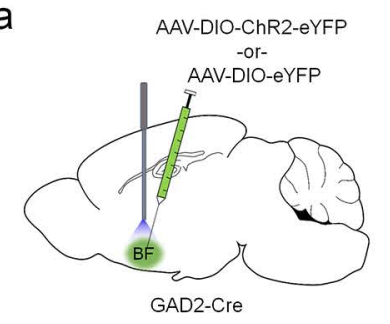

f

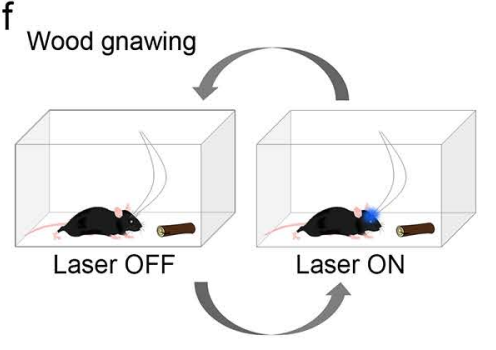

b

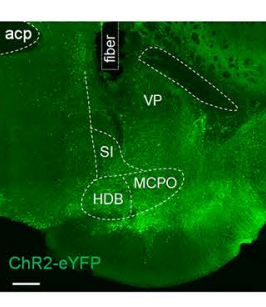

9

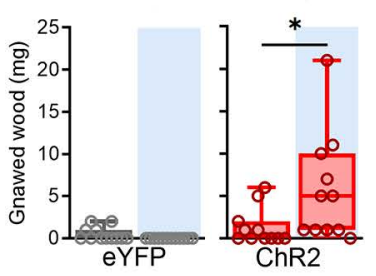

C

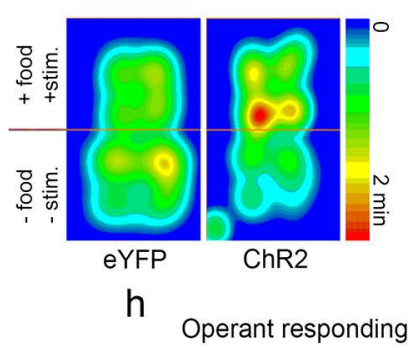

d

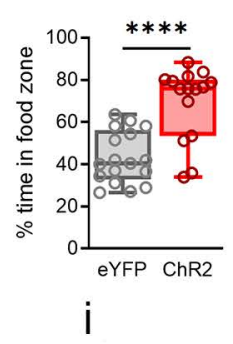

e

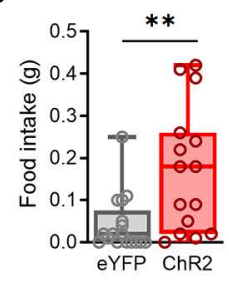

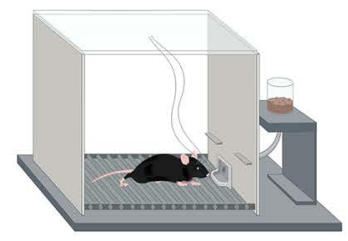

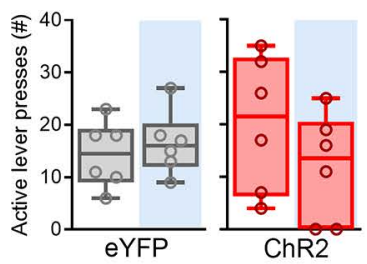

Figure 1. Activation of $\mathrm{BF}^{\mathrm{GAD2+}}$ neurons increases consummatory drive.

a, Stereotaxic targeting of viral vectors and optogenetic stimulation. b, Confocal image of viral expression and optic fiber placement in BF, $200 \mu \mathrm{m}$ scale bar. c, Heatmap of center body location, mean of eYFP and ChR2 groups, during test of food exposure paired with optic stimulation. One side of the arena contained food pellets, whereupon entry triggered optic stimulation. d, Percent time spent in food zone. Mann-Whitney test, $U=36,{ }^{* * *} p<0.001$. ChR2, $n=15 ;$ eYFP, $n=17$. e, Food intake, ingested mass. Mann-Whitney test, $U=54.5,{ }^{* *} p<0.01$. ChR2, $n=15$; eYFP $n=17$. $\mathbf{f}$, Design for test of wood gnawing behavior, wherein animals were exposed to a willow tree branch with and without laser stimulation, in a counterbalanced fashion. g. Amount of wood removed due to gnawing during laser ${ }^{\mathrm{N}}$ vs laser $^{\mathrm{OFF}}$ epochs. ChR2, Wilcoxon signed rank test, $W=44,{ }^{*} p<0.05$. ChR2, $n=11$; eYFP, $n=12$. $\mathbf{h}$, Design of test for operant responding for food. Task entails discrimination between two levers, only one of which upon pressing will result in food delivery. $\mathbf{i}$, Active lever presses during laser ${ }^{\mathrm{N}}$ vs laser ${ }^{\mathrm{OFF}}$ epochs. ChR2, $\mathrm{n}=6$; eYFP, $\mathrm{n}=6$. 
a

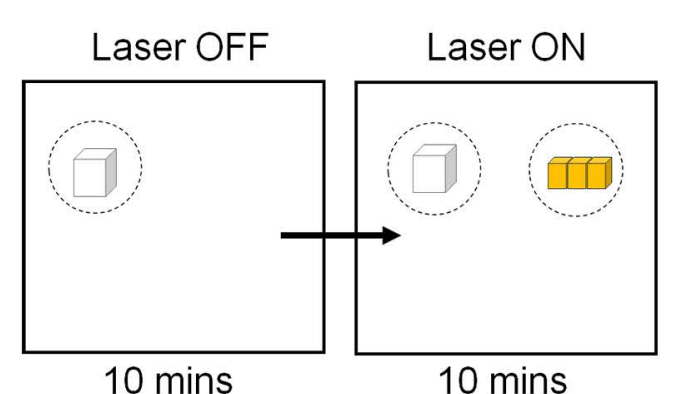

10 mins

10 mins

d

\section{Conditioning}
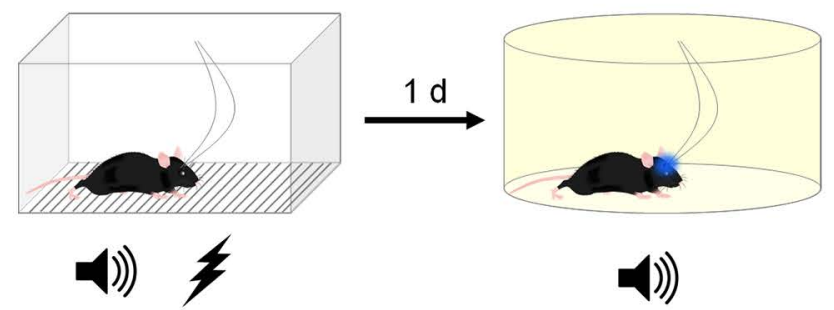

(4)) b

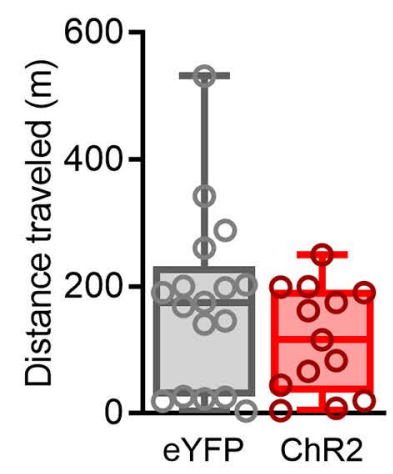

C

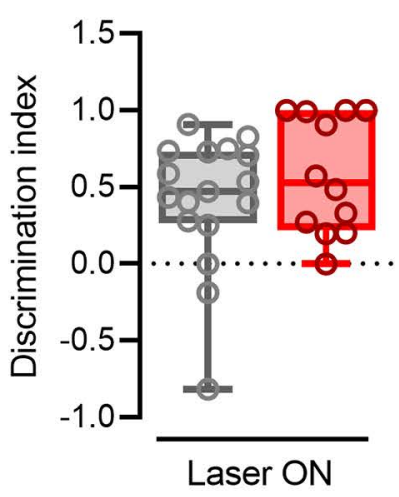

e

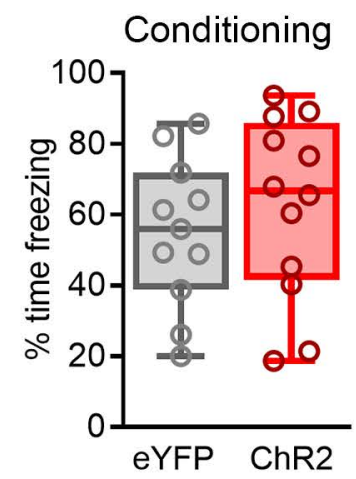

f

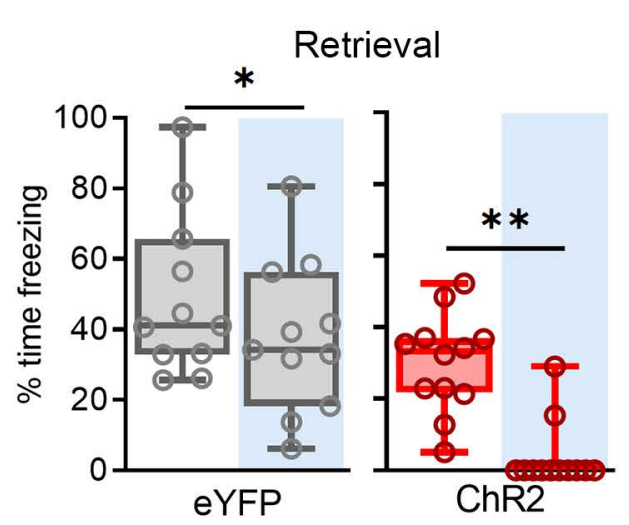

g

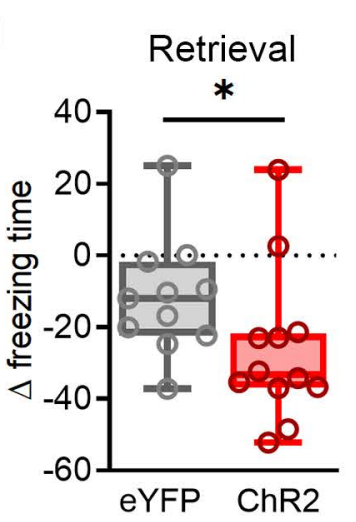

counterbalanced

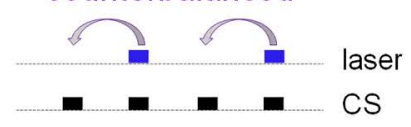

test trials

h

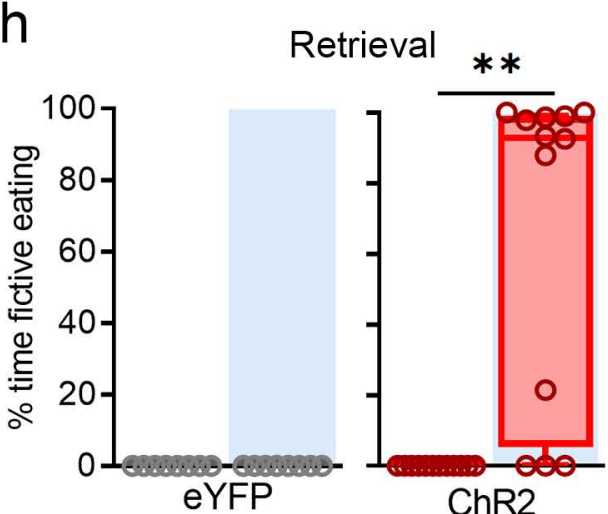

Figure 2. $\mathrm{BF}^{\mathrm{GAD2}+}$ neuron activation does not affect exploration or object recognition but disrupts conditioned fear expression.

$\mathbf{a}$, Design of novel object recognition test. $\mathbf{b}$, Discrimination index is measured as the time spent exploring the novel object - time spent exploring the familiar object divided by the total time exploring novel object and familiar object. ChR2, $\mathrm{n}=12$; eYFP, $n=17$. c, Total distance traveled during laser ${ }^{\mathrm{ON}}$ epoch. ChR2, $n=12$; eYFP, $n=17$. d, Design for test of fear memory expression. Following conditioning in context A, animals were placed into a distinct arena (context B) and presented with $4 \mathrm{CS}$ trials, two of which coincided with photostimulation, in a counterbalanced fashion. e, Freezing levels during final two CS trials of conditioning. $f$, CS-evoked freezing during laser ${ }^{\mathrm{ON}}$ vs. laser ${ }^{\mathrm{OFF}} \mathrm{CS}$ trials. ChR2, Wilcoxon signed rank test, $W=-66,{ }^{* *} \mathrm{p}<0.01$. eYFP, paired t-test, $\mathrm{t}_{10}=2.40,{ }^{*} \mathrm{p}<0.05$. ChR2, $\mathrm{n}=12 ; \mathrm{eYFP}, \mathrm{n}=11$. $\mathrm{g}$, Difference in freezing during laser ${ }^{\mathrm{ON}}$ vs. laser ${ }^{\mathrm{OFF}} \mathrm{CS}$ trials. ChR2, $n=12$; eYFP, $n=11$. $\mathbf{h}$, Fictive eating during laser ${ }^{\mathrm{ON}}$ vs. laser $^{\mathrm{OFF}}$ CS trials. ChR2, Wilcoxon signed rank test, $W=45,{ }^{* *} p<0.01$. 
a

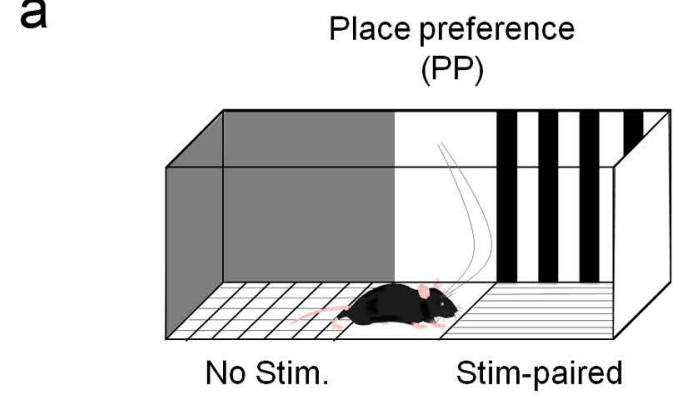

C

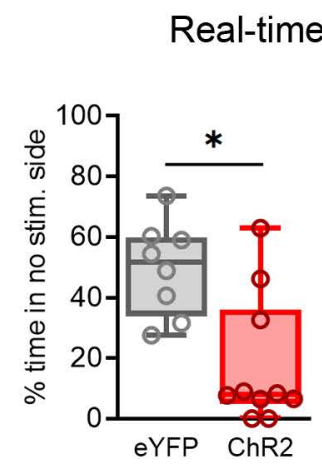

Place preference

(PP)

PP (RT-PP)

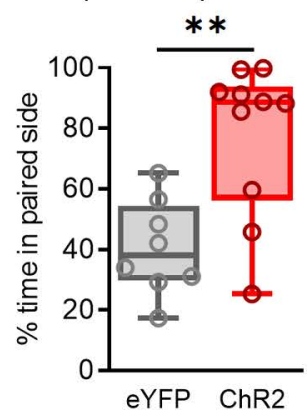

b

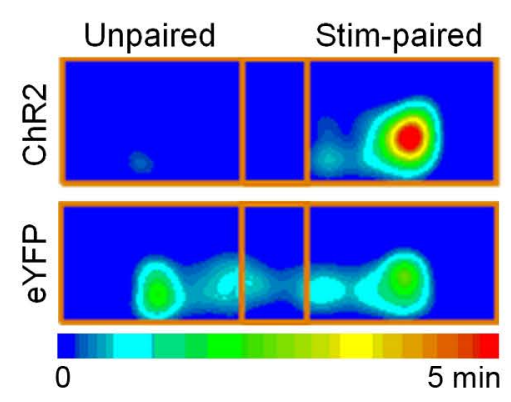

Conditioned PP (CPP)

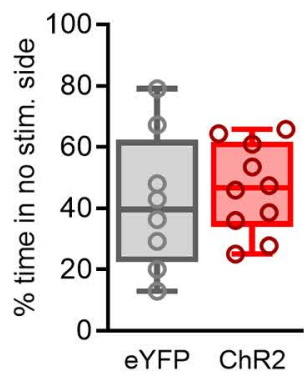

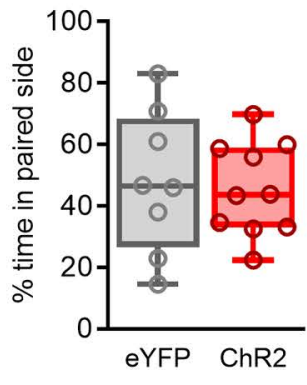

Figure 3. Reward-like effect of $\mathrm{BF}^{\mathrm{GAD} 2+}$ activation.

$\mathbf{a}$, Design for test of real-time and conditioned place preference. $\mathbf{b}$, Heatmap of center body location, of ChR2 and eYFP sample animal. ChR2, $n=10$; eYFP, $n=8$. c, Real-time place preference, measured on day 1 during photostimulation. Percent time in unpaired side, Mann-Whitney test, $U=12$, ${ }^{*} p<0.05$. Percent time in stimulation paired side, MannWhitney test, $U=10,{ }^{*} p<0.01$. ChR2, $n=10$; eYFP, $n=8$. d, Conditioned place preference, measured on day 2 in the absence of photostimulation. ChR2, $n=10$; eYFP, $n=8$. 
a

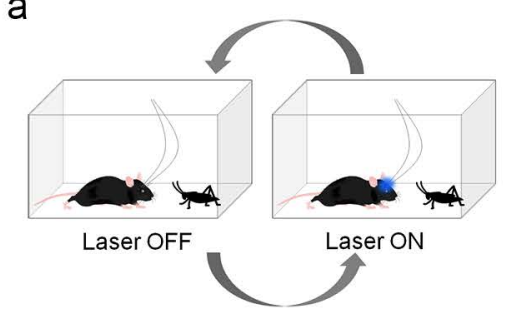

e

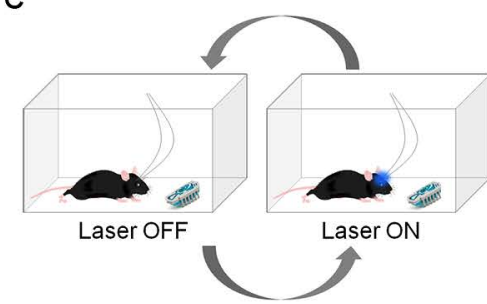

i

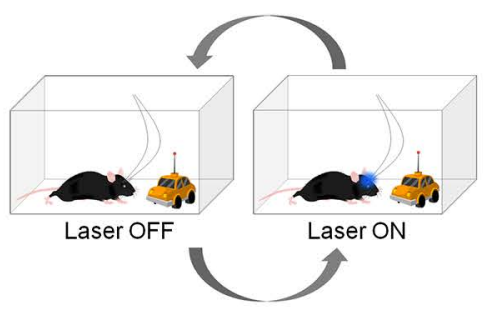

b

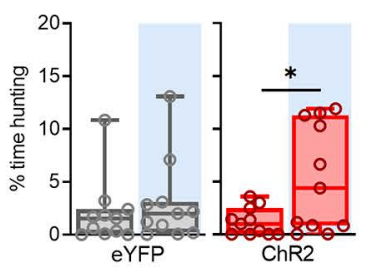

f

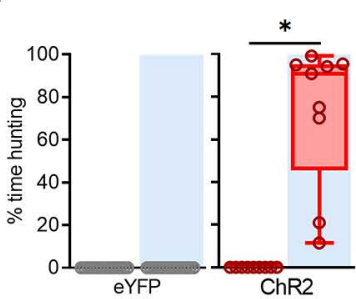

j

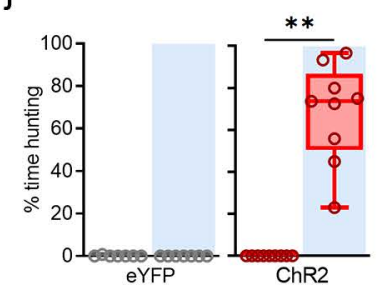

$c$

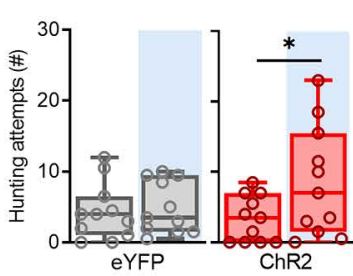

9

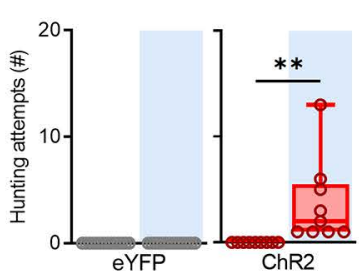

k

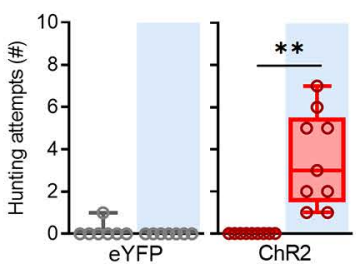

d

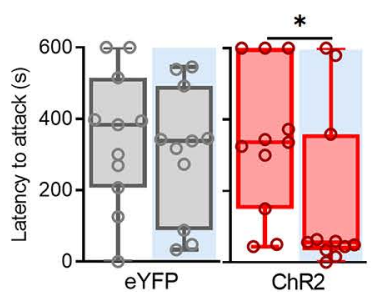

h

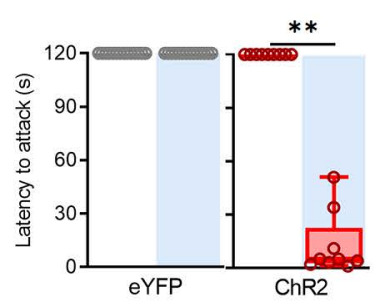

।

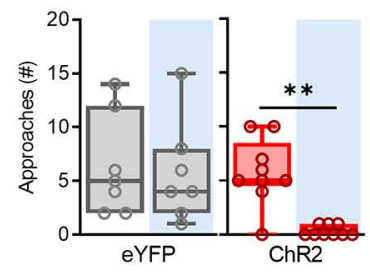

Figure 4. $\mathrm{BF}^{\mathrm{GAD2+}}$ activation promotes hunting of live and artificial prey.

$\mathbf{a}$, Design of live cricket prey hunting assay used in $\mathbf{b}$-d. $\mathbf{b}$, Percent time hunting, defined as the time spent pursuing and capturing the cricket. ChR2, Wilcoxon signed rank test, $W=43,{ }^{*} p<0.05$. ChR2, $\mathrm{n}=11$; eYFP, $\mathrm{n}=11$. c, Number of hunting attempts. ChR2, paired t-test, $\mathrm{t}_{10}=2.36,{ }^{*} \mathrm{p}<0.05$. ChR2, $\mathrm{n}=11$, eYFP, $\mathrm{n}=11$. d, Latency to attack. ChR2, Wilcoxon signed rank test, $W=-43,{ }^{*} p<0.05$. e, Design for artificial prey hunting assay used in f-h. Artificial prey consisted of a small toy (robobug) that is propelled by vibration. f, Percent time hunting. ChR2, Wilcoxon signed rank test, $W=45,{ }^{* *} \mathrm{p}<0.01$. ChR2, $\mathrm{n}=9$; eYFP, $\mathrm{n}=14$. g, Number of hunting attempts. ChR2, Wilcoxon signed rank test, $W=45$, ${ }^{* *} p<0.01$. h, Latency to attack. ChR2, Wilcoxon signed rank test, $W=45,{ }^{* *} p<0.01$. i, Design of the interactive artificial prey assay used in $\mathbf{j}-\mathbf{l}$. Interactive prey consisted of a toy car remote-controlled by the experimenter. $\mathbf{j}$, Percent time hunting. ChR2, Wilcoxon signed rank test, $W=45$, ${ }^{* *} p<0.01$. ChR2, $n=9$; eYFP, $n=7$. k, Number of hunting attempts. ChR2, Wilcoxon signed rank test, $W=45,{ }^{* *} \mathrm{p}<0.01$. ChR2, $\mathrm{n}=9$; eYFP, $\mathrm{n}=7$. I, Latency to attack. ChR2, Wilcoxon signed rank test, $W=45,{ }^{* *} \mathrm{p}<0.01$. ChR2, $\mathrm{n}=9$; eYFP, $\mathrm{n}=7$. 
a

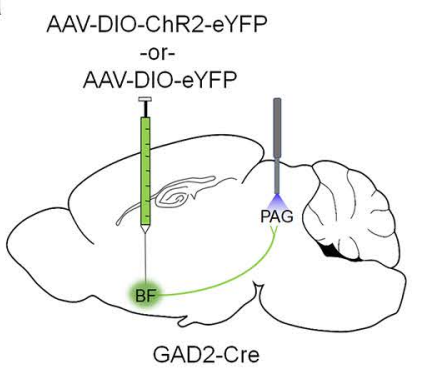

f

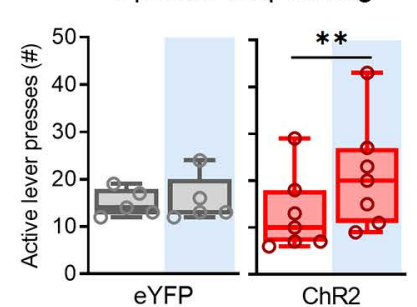

j

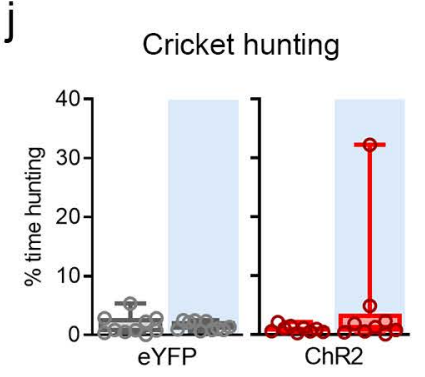

b

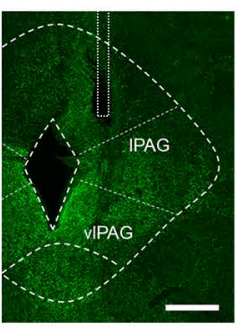

g

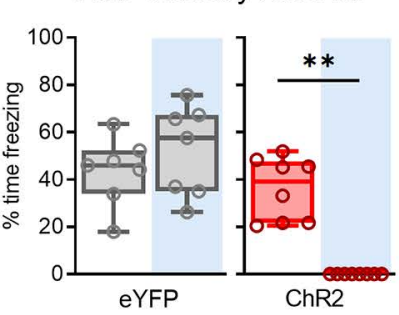

k

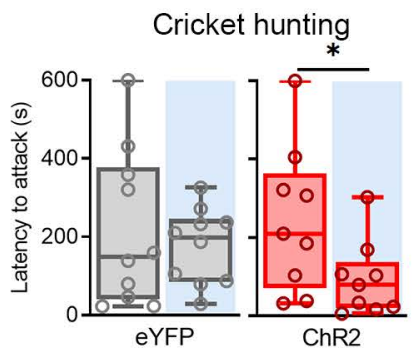

$\mathrm{h}$ d

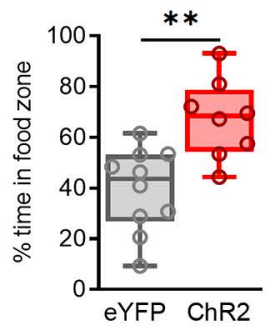

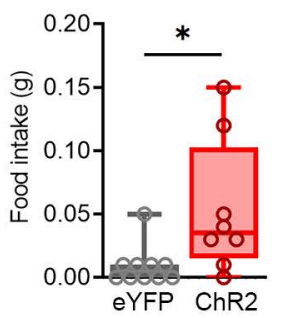

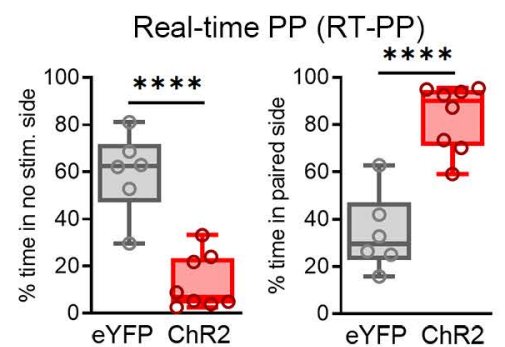

I

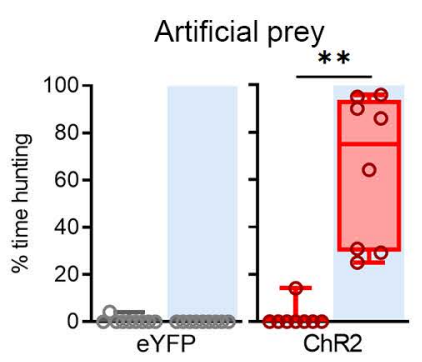

e

Wood gnawing

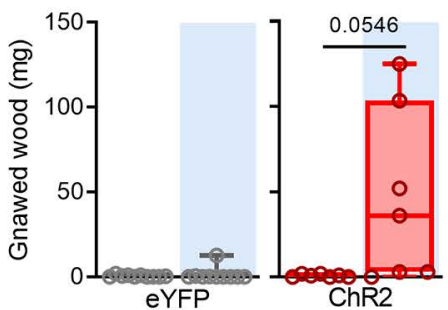

i

Conditioned PP (CPP)

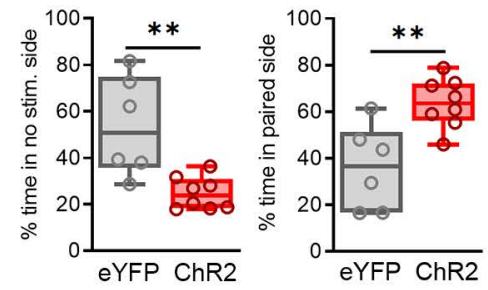

m

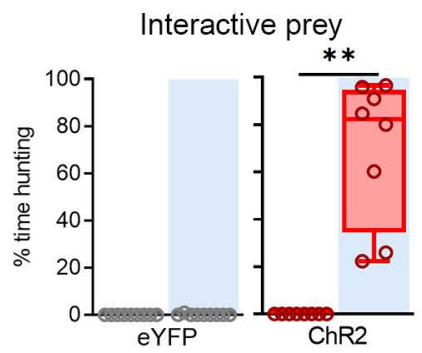

Figure 5. PAG projections mediate BF effects on consumption, reinforcement and predatory hunting.

a, Stereotaxic targeting of viral vectors and optogenetic stimulation. $\mathbf{b}$, Confocal image of viral expression and optic fiber placement in BF, $200 \mu \mathrm{m}$ scale bar. c, Percent time spent in food zone during optic stimulation paired with food. MannWhitney test, $U=14,{ }^{*} p<0.05$. ChR2, $n=8$; eYFP, $n=10$. d, Food intake, ingested mass. Unpaired t-test, $\mathrm{t}_{16}=3.66$. ${ }^{* *}$ $p<0.01$. ChR2, $n=8$; eYFP, $n=10$. e, Amount of wood removed due to gnawing during laser ${ }^{O N}$ vs laser ${ }^{O F F}$ epochs. ChR2, paired t-test, $t_{6}=2.38$. ChR2, $n=7$; eYFP, $n=10$. $f$, Active lever presses during test of operant responding for food. ChR2, paired t-test, $t_{6}=3.78,{ }^{*} p<0.05$. ChR2, $n=7$; eYFP, $n=5$. g, CS-evoked freezing during laser ${ }^{\mathrm{ON}}$ vs. laser ${ }^{\mathrm{OFF}} \mathrm{CS}$ trials, following the experimental design in Fig. 2d. ChR2, Wilcoxon signed rank test, $\mathrm{W}=-36,{ }^{* *} p<0.01$. ChR2, $\mathrm{n}=8$; eYFP, $\mathrm{n}=7$. $\mathbf{h}$, Real-time place preference, measured on day 1 during photostimulation. Percent time in unpaired side, unpaired $\mathrm{t}$-test, $\mathrm{t}_{12}=6.03, * * * \mathrm{p}<0.0001$. Percent time in stimulation paired side, paired $\mathrm{t}$-test, $\mathrm{t}_{12}=6.06$, $* * * * \mathrm{p}<0.0001$. ChR2, $\mathrm{n}=8$; eYFP, $n=6$. i, Conditioned place preference, measured on day 2 in the absence of photostimulation. Percent time in unpaired side, paired t-test, $\mathrm{t} 12=3.62,{ }^{* *} \mathrm{p}<0.01$. Percent time in stimulation paired side, paired t-test, $\mathrm{t}_{12}=3.62$, ${ }^{* *} \mathrm{p}<0.01$. ChR2, $\mathrm{n}=8$; eYFP, $\mathrm{n}=6$. $\mathrm{j}$, Percent time spent hunting in the live cricket assay. ChR2, $n=9$; eYFP, $n=10$. $\mathbf{k}$, Latency to attack in the live cricket assay. ChR2, paired t-test, $t_{8}=2.82,{ }^{*} p<0.05$. ChR2, $n$ $=9 ;$ eYFP, $n=10$. I, Percent time hunting in the artificial prey assay. ChR2, Wilcoxon signed rank test, $W=36,{ }^{* *} p<0.01$. ChR2, $n=8$; eYFP, $n=9$. $m$, Percent time hunting in the interactive artificial prey assay. ChR2, Wilcoxon signed rank test, $W=36,{ }^{* *} \mathrm{p}<0.01$. ChR2, $\mathrm{n}=8$; eYFP, $\mathrm{n}=9$. 


\section{Supplementary Files}

This is a list of supplementary files associated with this preprint. Click to download.

- RomanOrtizetal.2021SupplementaryFigures.pdf

- SupplementaryVideo1.mp4

- SupplementaryVideo2.mp4

- SupplementaryVideo3.mp4 